Int. J. Dev. Biol. 59: 217-219 (2015)

doi: $10.1387 / \mathrm{ijdb} .150025 \mathrm{dr}$

\title{
A simple method of image analysis to estimate CAM vascularization by APERIO ImageScope software
}

\author{
CHRISTIAN MARINACCIO ${ }^{1}$ and DOMENICO RIBATTI ${ }^{*, 1,2}$ \\ ${ }^{1}$ Department of Basic Medical Sciences, Neurosciences, and Sensory Organs, University of Bari Medical School and \\ ${ }^{2}$ National Cancer Institute "Giovanni Paolo II", Bari, Italy
}

\begin{abstract}
The chick chorioallantoic membrane (CAM) assay is a well-established method to test the angiogenic stimulation or inhibition induced by molecules and cells administered onto the CAM. The quantification of blood vessels in the CAM assay relies on a semi-manual image analysis approach which can be time consuming when considering large experimental groups. Therefore we present here a simple and fast volumetric method to inspect differences in vascularization between experimental conditions related to the stimulation and inhibition of CAM angiogenesis based on the Positive Pixel Count algorithm embedded in the APERIO ImageScope software.
\end{abstract}

KEY WORDS: chick chorioallantoic membrane assay, image analysis, angiogenesis

\section{Introduction}

The formation of new blood vessels from pre-existing ones, commonly referred as angiogenesis, is a process occurring in both normal and pathologic conditions, including embryonic development, ovulation, wound healing, and cancer (Ribatti et al., 2007a). Among the various in vivo assays developed for the study of the angiogenesis, the chick embryo chorioallanotoic membrane (CAM) represents one of the most reliable tools for the study of the effects of angiogenic or anti-angiogenic chemical and biological molecules on neovascularization. Moreover, the CAM assay have been extensively employed in cancer research to assess the angiogenic potential of cancer cells from a variety of malignancies and the inhibition of the cancer cells-generated angiogenic response using anti-angiogenic molecules (Park et al., 2009, Ria et al., 2002, Ribatti et al., 2007b, Ribatti et al., 2002, Vacca et al., 2003). The advantages of this assay includes simple processing and maintenance of the chick embryos, short experimental times and low costs and the possibility to exploit the relative immature immune system of the chick embryo at the early stages of embryonic development, allowing mammalian cell xenografts and reducing the occurrence of non-specific inflammatory responses.

Counting of blood vessels has been the preferred method to quantify the angiogenic response occurring after the exposure to the angiogenic stimulus, achieved by counting the number of vessels radially converging toward the implant under a stereomicroscope. The data from an experimental group can then be expressed as the mean of the vessels counted per egg $\pm 1 \mathrm{~s}$. $\mathrm{d}$. This approach is often time consuming, especially with large experimental conditions. Image analysis tools are increasingly being used to shorten experimental analysis times and provide a reliable and standardized quantification in terms of pixel intensities, defining a volumetric rather than a counting approach to find significant differences in angiogenic responses compared to controls. In this technical note, we present a simple volumetric approach to the study of angiogenesis induced in the CAM assay using the Positive Pixel Count algorithm embedded in the Aperio ImageScope software to assess differences between CAM treated with an angiogenic stimulus, CAM treated with an anti-angiogenic stimulus and CAM treated with culture medium alone as a negative control.

\section{Experimental Protocols}

\section{In vivo angiogenesis CAM assay}

Fertilized White Leghorn chicken eggs (30 per group) were incubated at $37^{\circ} \mathrm{C}$ at constant humidity. On day 3 , a square window was opened in the shell, and 2 to $3 \mathrm{ml}$ of albumen were removed to allow detachment of the developing CAM from the shell. The window was sealed with a glass, and the eggs returned to the incubator. On day 8, eggs were treated with $1 \mathrm{~mm}^{3}$ sterilized gelatin sponges (Gelfoam Upjohn Company, Kalamazoo, MI, USA) placed on the top of the growing CAM, as previously described (Ribatti et al., 2006). The sponges were then loaded with human recombinant

Abbreviations used in this paper: CAM, chick chorioallantoic membrane.

\footnotetext{
*Address correspondence to: Domenico Ribatti. Department of Basic Medical Sciences, Neurosciences, and Sensory Organs, University of Bari Medical School, Piazza Giulio Cesare, 11, 70124 Bari, Italy.Tel: +39-080-5478326. Fax: +39-080-5478310. E-mail: domenico.ribatti@uniba.it
}

Accepted: 16 April 2015.

ISSN: Online 1696-3547, Print 0214-6282 


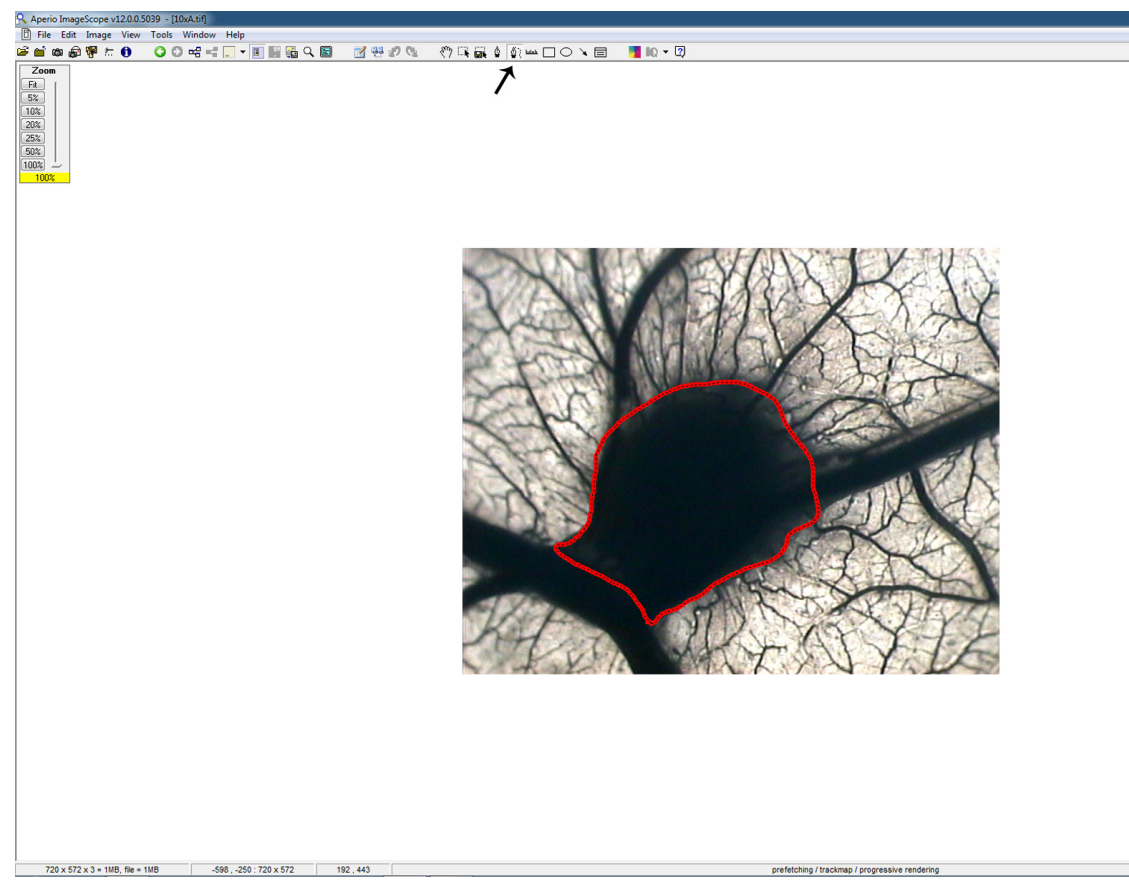

Fig. 1 (Left). Exclusion of the gelatin sponge area from the analysis through the negative pen tool.

Fig. 2 (Right). Parameters of the Positive Pixel Count algorithm.

vascular endothelial growth factor $\left(\right.$ hrVEGF $_{165}$ ) (R\&D System, Abingdon, UK) at $500 \mathrm{ng} / \mathrm{sponge}$ as an angiogenic stimulus, or with $1.75 \mu \mathrm{mol} / \mathrm{L} / \mathrm{sponge}$ lenalidomide as an angiogenic inhibitor, or with $1 \mu \mathrm{l} /$ sponge RPMI-1640 as a negative control. CAMs were examined daily until day 12 and photographed in ovo with a stereomicroscope equipped with a camera (Olympus Italia, Rozzano, Italy) at 50X magnification.

\section{Image analysis}

Microscopic images obtained from the stereomicroscope were converted in gray-scale and analyzed using the Aperio Positive

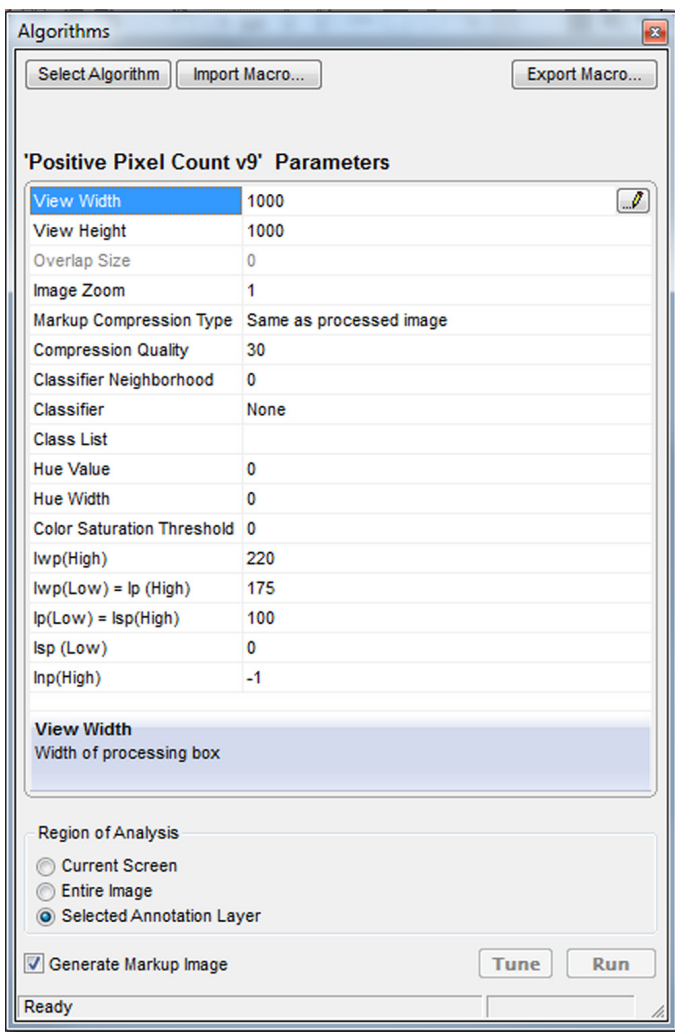

Pixel Count algorithm embedded in the ImageScope v.11.2.0.780 (Leica Biosystems, Nussloch, Germany). All the images were analyzed with the exclusion of the gelatin sponge area using the negative pen tool to eliminate non-specific detection in this area as shown in Fig. 1. The algorithm input parameters (Fig. 2) were initially set to obtain the identification of pixels related to the blood vessels as strong positive and to the background as medium and weak positive and tuned to minimize non-specific pixel recognition as strong positive.

The algorithm output is composed of the number of strong positive pixels (Nsp), the number of medium positive pixels ( $\mathrm{Np}$ ),

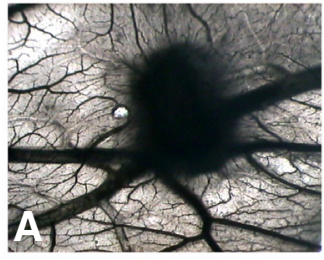

ANGIOGENIC STIMULUS

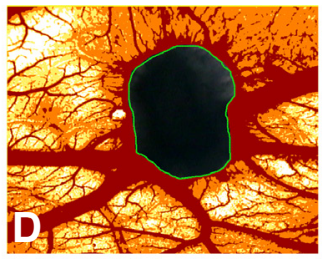

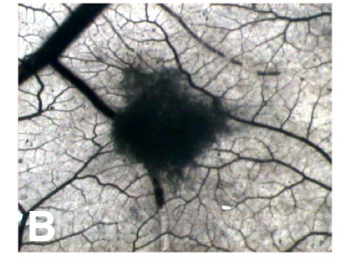

CONTROL

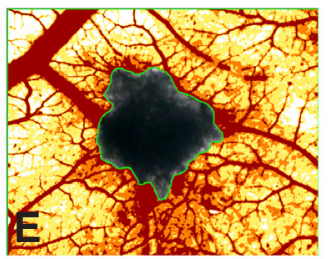

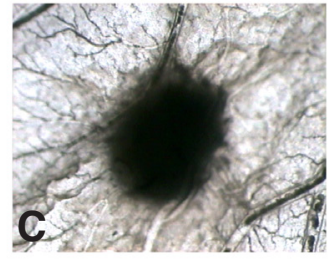

ANGIOGENIC INHIBITION

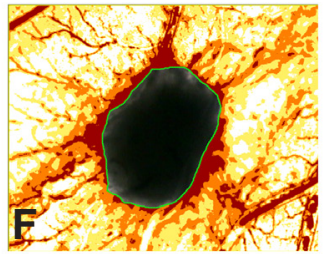

G

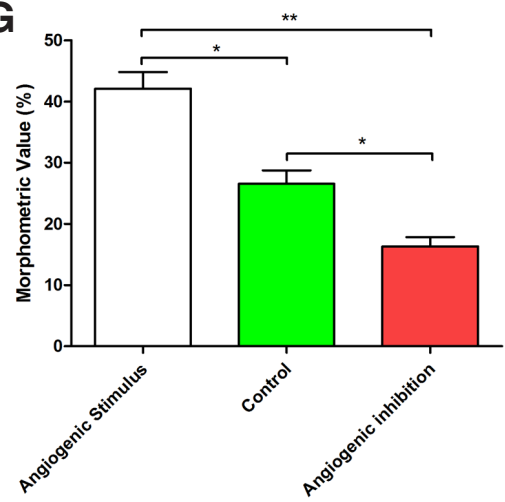

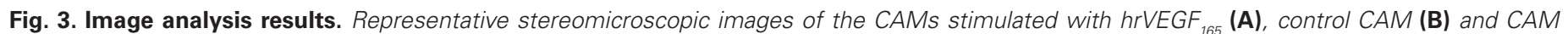
inhibited with lenalidomide (C). Mark-up images of stimulated (D), control (E) and inhibited (F) CAMs. Comparison between the morphometric values obtained from the analysis of images from CAMs treated with hrVEGF ${ }_{165}$, control and lenalidomide with a significant difference between the experimental conditions (G). ${ }^{*} p<0.05 ;{ }^{* *} p<0.01$. Mark-up image color code. Red: strong positive pixels; Orange: medium positive pixels; Yellow: weak positive pixels. 
the number of weak positive pixels (Nwp).

A morphometric value is then defined and calculated by the algorithm as:

$$
\text { Number of strong positive pixels }(\%)=\frac{N s p}{N p+N w p+N s p} \times 100
$$

to establish a comparison between the experimental groups treated with the angiogenic stimulus, angiogenic inhibitor, or medium alone.

\section{Statistical analysis}

Statistical significance between the stimulated, normal and inhibited experimental groups was assessed by one-way Anova followed by Tukey multiple comparison post-test. The statistical analysis and graph plotting were performed with the Graph Pad Prism 5.0 statistical package (GraphPadSoftware, San Diego, CA, USA).

\section{Results and Discussion}

\section{Image analysis reveals significant differences between the} experimental conditions

CAMs treated with the angiogenic stimulus, angiogenic inhibitor, or medium alone revealed a significant difference in the percentage of strong positive pixels which identifies the blood vessels ramifications (Table 1) In detail, CAMs treated with VEGF showed the highest percentage of strong positive pixels (mean $=42.11 \% \mathrm{SD}=$ 2.70 ) when compared to the control (mean $=26.55 \% \mathrm{SD}=2.19$ ) and lenalidomide treated CAMs (mean $=16.35 \% \mathrm{SD}=1.48$ ), while the CAMs treated with lenalidomide showed the lowest percentage of strong positive pixels when compared with the control and VEGF treated CAMs (Fig. 3).

Blood vessel development through angiogenesis has been considerably and extensively studied in a range of normal and pathologic conditions, with a particular attention on its role on cancer progression, predicted more than 40 years ago by Judah Folkman and established as an hallmark of tumor growth (Ribatti, 2014). A variety of assays have been developed to investigate the stimulation or inhibition effects of pro- and anti-angiogenic molecules as well as cells and tissues on angiogenesis. Among them, the CAM assay is one of the most diffuse techniques for observing blood vessel angiogenesis. The quantification of the angiogenic response on microscopic images still relies on semimanual analysis of the regions of interest, which can be a time consuming approach especially with large samples.

In this report we have highlighted the reliability of a volumetric approach based on the percentage of pixels that identifies blood vessels using the Positive Pixel Count algorithm embedded in
TABLE 1

\section{MEASUREMENT DATA}

\begin{tabular}{cccc} 
CAM \#* $^{*}$ & Stimulus applied & Mean Morphometric value (\%) & Standard Deviation \\
\hline $1-30$ & hrVEGF $_{165}$ & $42.11 \%$ & 2.70 \\
$31-60$ & RPMI-1640 & $26.55 \%$ & 2.19 \\
$61-90$ & Lenalidomide & $16.35 \%$ & 2.48 \\
\hline
\end{tabular}

${ }^{*}$ CAMs were grouped by the stimulus applied and mean morphometric data is shown.

the Aperio ImageScope software. The algorithm was successfully able to spot significant differences in three classical experimental conditions involving the stimulation, the inhibition and no treatment of the CAM angiogenesis, allowing a faster image processing and analysis compared to the semi-manual counting approach. A potential limit of this approach is the absence of information about other parameters such as blood vessel length, area and width, so this method should be used in association with other proposed automated quantitative analysis (Shi et al., 2014) or used as a fast inspection method to spot differences in CAM vascularization before a more detailed analysis.

\section{References}

PARK, B.C., PARK, S.Y., LEE, J.S., MOUSA, S.A., KIM, J.T., KWAK, M.K., KANG, K.W. LEE, E.S., CHOI, H.G., YONG, C.S. et al., (2009). The anti-angiogenic effects of 1-furan-2-yl-3-pyridin-2-yl-propenone are mediated through the suppression of both VEGF production and VEGF-induced signaling. Vascul Pharmacol 50: 123-131.

RIA, R., LOVERRO, G., VACCA, A., RIBATTI, D., CORMIO, G., ROCCARO, A.M and SELVAGGI, L. (2002). Angiogenesis extent and expression of matrix metalloproteinase-2 and -9 agree with progression of ovarian endometriomas. Eur $J$ Clin Invest 32: 199-206.

RIBATTI, D. (2014). History of research on angiogenesis. Chem Immunol Allergy 99: 1-14.

RIBATTI, D., NICO, B., CRIVELLATO, E., ROCCARO, A.M. and VACCA, A. (2007a). The history of the angiogenic switch concept. Leukemia 21: 44-52.

RIBATTI, D., NICO, B., MANGIERI, D., LONGO, V., SANSONNO, D., VACCA, A. and DAMMACCO, F. (2007b). In vivo inhibition of human hepatocellular carcinoma related angiogenesis by vinblastine and rapamycin. Histol Histopathol22: 285-289.

RIBATTI, D., NICO, B., VACCA, A. and PRESTA, M. (2006). The gelatin spongechorioallantoic membrane assay. Nat Protoc 1: 85-91.

RIBATTI, D., RAFFAGHELLO, L., PASTORINO, F., NICO, B., BRIGNOLE, C., VACCA, A. and PONZONI, M. (2002). In vivo angiogenic activity of neuroblastoma correlates with MYCN oncogene overexpression. Int J Cancer 102: 351-354.

SHI, P., HONG, J., HUANG, Y., ZHANG, Z., ZHANG, M. and ZHANG, L. (2014) Automated computational framework of blood vessel quantification in chick chorioallantoic membrane angiogenesis. J Biomed Opt 19: 106005.

VACCA, A., RIA, R., SEMERARO, F., MERCHIONNE, F., COLUCCIA, M., BOCCARELLI, A., SCAVELLI, C., NICO, B., GERNONE, A., BATTELLI, F. et al., (2003). Endothelial cells in the bone marrow of patients with multiple myeloma. Blood 102: $3340-3348$ 


\section{Further Related Reading, published previously in the Int. J. Dev. Biol.}

Differential expression of angiogenic and anti-angiogenic molecules in the chick embryo chorioallantoic membrane and selected organs during embryonic development

Christian Marinaccio, Beatrice Nico and Domenico Ribatti

Int. J. Dev. Biol. (2013) 57: 907-916

http://dx.doi.org/10.1387/ijdb.130317dr

Zebrafish embryo, a tool to study tumor angiogenesis

Chiara Tobia, Giulia De Sena and Marco Presta

Int. J. Dev. Biol. (2011) 55: 505-509

http://dx.doi.org/10.1387/ijdb.103238ct

The use of the orthotopic model to validate antivascular therapies for cancer

Monica Loi, Daniela Di Paolo, Pamela Becherini, Alessia Zorzoli, Patrizia Perri, Roberta Carosio, Michele Cilli, Domenico Ribatti, Chiara Brignole, Gabriella Pagnan, Mirco Ponzoni and Fabio Pastorino

Int. J. Dev. Biol. (2011) 55: 547-555

http://dx.doi.org/10.1387/ijdb.103230ml

\section{Tumor blood vessel visualization}

Jeannine Missbach-Guentner, Julia Hunia and Frauke Alves

Int. J. Dev. Biol. (2011) 55: 535-546

http://dx.doi.org/10.1387/ijdb.103229jm

Role of laminins in physiological and pathological angiogenesis

Patricia Simon-Assmann, Gertraud Orend, Elmina Mammadova-Bach, Caroline Spenlé and Olivier Lefebvre

Int. J. Dev. Biol. (2011) 55: 455-465

http://dx.doi.org/10.1387/ijdb.103223ps

Paracrine regulation of angiogenesis by different cell types in the aorta ring model Roberto F. Nicosia, Penelope Zorzi, Giovanni Ligresti, Ann Morishita and Alfred C. Aplin Int. J. Dev. Biol. (2011) 55: 447-453

http://dx.doi.org/10.1387/ijdb.103222rn

A brief history of angiogenesis assays

Anca-Maria Cimpean, Domenico Ribatti and Marius Raica

Int. J. Dev. Biol. (2011) 55: 377-382

http://dx.doi.org/10.1387/ijdb.103215ac

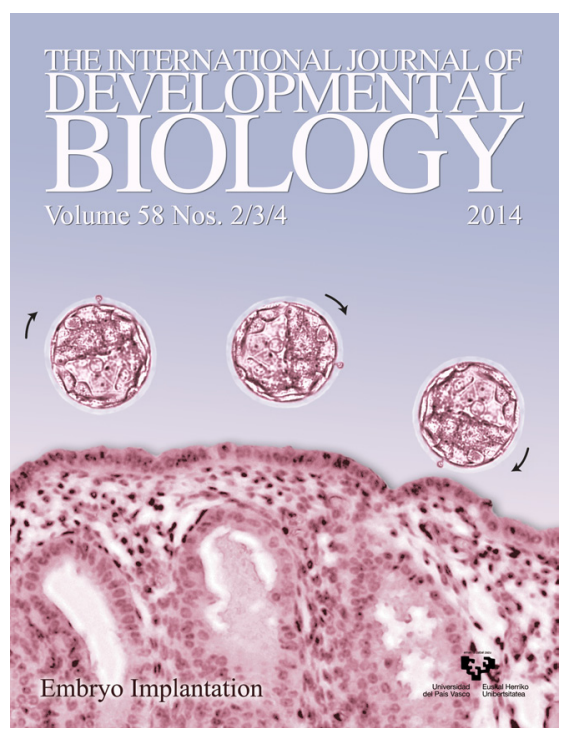

5 yr ISI Impact Factor $(2013)=2.879$

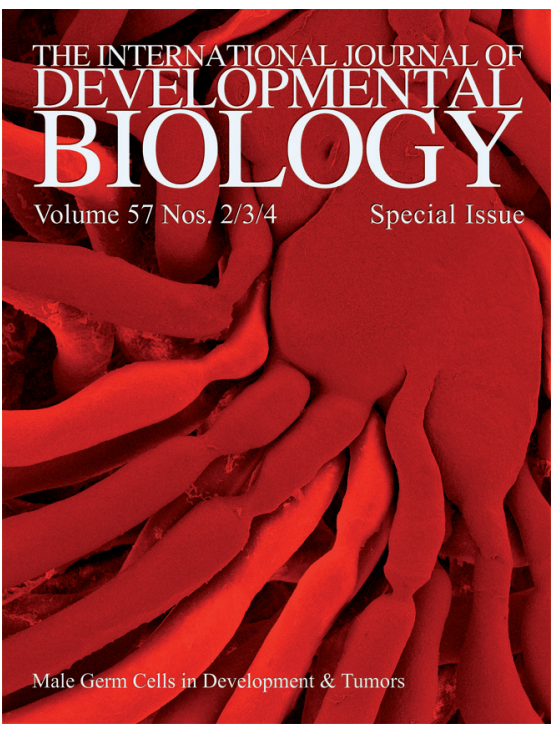

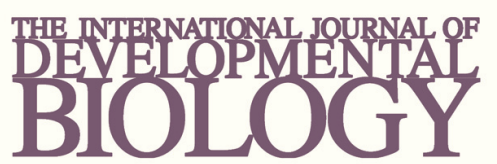

Volume 54 Nos. 6/7

Special Issue
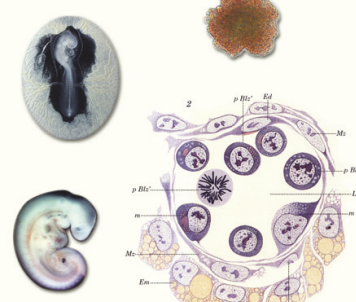

(2)
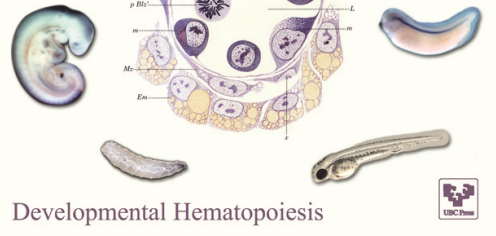

Developmental Hematopoiesis
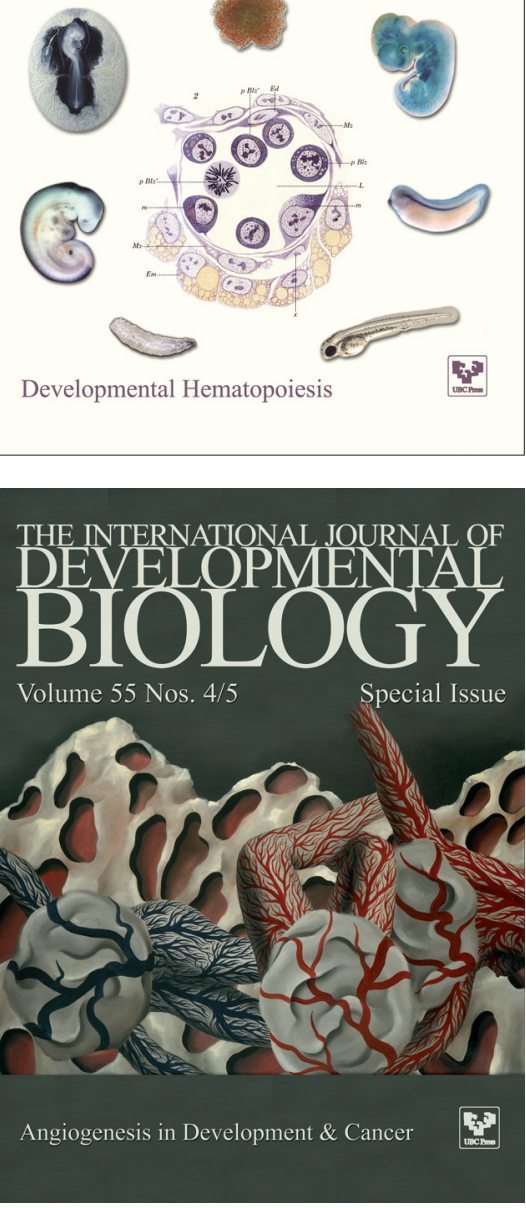